\title{
Sur l'existence et l'unicité de la solution d'une équation différentielle à argument retardé
}

\author{
par J. BEAż (Katowice)
}

1. Dans cette note j'établis un théorème d'existence et un critère d'unicité pour la solution de l'équation différentielle à argument retardé de la forme:

$$
\begin{aligned}
& \varphi(t)=\omega(t) \quad \text { pour } t \leqslant 0, \\
& \varphi^{\prime}(t)=\int_{0}^{\infty} f[t, \varphi(t-s)] d r(t, s)+q(t) \quad \text { pour } \quad t \in \Delta=\langle 0, a\rangle .
\end{aligned}
$$

L'équation (1) correspond à un phénomène dans lequel la vitesse du processus à un instant donné dépend de l'état de ce processus dans un intervalle de temps antérieur à l'instant donné. A. D. Myszkis [3] a démontré un théorème sur l'existence et l'unicité des solutions d'équations linéaires à argument retardé dans le cas où l'intervalle de temps considéré est fini. Dans le cas d'un intervalle infini, un théorème d'existence a été donné pour les équations non-linéaires à argument retardé par $\mathbf{A}$. Bielecki et M. Maksym [1].

J'exprime ma vive reconnaissance à M. A. Bielecki qui a bien voulu m'assister de ses conseils ou cours de la rédaction de cette note; je remercie aussi M. Z. Opial dont les précieuses remarques m'ont permis d'améliorer la rédaction définitive.

2. Nous admettrons pour l'équation (1) les hypothèses suivantes:

Hypothises (H). $1^{\circ}$ La fonction $f(t, x)$ est définie et continue dans le domaine $D=\{(t, x): 0 \leqslant t \leqslant a,-\infty<x<+\infty\}$.

$2^{\circ}$ La fonction initiale $\omega(t)$ est continue et bornée dans l'intervalle $(-\infty, 0)$.

$3^{\circ}$ Le noyau $r(t, s)$ est une fonction, définie pour $t \geqslant 0$ et $s \geqslant 0$, et on $a r(t, 0) \equiv \mathbf{0}$.

$4^{0}$ La variation de la fonction $r(t, s)$ par rapport à la variable $s$ satisfait pour tout $t \in \Delta$ à l'inégalité $\bigvee_{s=0}^{\infty} r(t, s) \leqslant V=$ const. 
$5^{\circ}$ Quel que soit $\varepsilon>0$ il existe un nombre $K>0$ tel que $\bigvee_{s=K}^{\infty} r(t, s)<\varepsilon$ pour tout $t \in \Delta$.

$6^{\circ}$ Quels que soient les nombres $k>0$ et $u \in \Delta$ il existe

$$
\lim _{t \rightarrow u} \int_{0}^{k}|r(t, s)-r(u, s)| d s=0, \quad t \in \Delta .
$$

Moyennant les hypothèses $(\mathbf{H})$ on peut démontrer (v. [1]) un lemme sur la continuité de l'intégrale de Stieltjes

$$
\text { - } \Theta(t)=\int_{0}^{\infty} f[t, \varphi(t-s)] d r(t, s) .
$$

LEMмE. Si la fonction $\varphi(t)$ est continue et bornée dans l'intervalle $(-\infty, a\rangle$, la fonction $\Theta(t)$, définie par la formule (2), est continue dans l'intervalle $\Delta$.

En vu de démontrer le théorème d'existence, supposons réalisées, outre les hypothèses $(\mathbf{H})$, les hypothèses $\left(\mathbf{H}^{*}\right)$ suivantes:

HyPothises ( $\left.\mathrm{H}^{*}\right)$. $1^{*}$ Pour tout point du domaine $D$ on a les inégalités $|f(t, x)| \leqslant \Phi(t,|x|)$, où la fonction $\Phi(t, y)$ est continue et non négative dans le domaine $D_{+}=\{(t, y): 0 \leqslant t \leqslant a, y \geqslant 0\}$, non décroissante par rapport à la variable $y$.

2* Toutes les intégrales supérieures de l'équation différentielle sans retard $y^{\prime}(t)=V \Phi(t, y)$, où le nombre $V$ est défini dans l'hypothèse $4^{\circ}(\mathbf{H})$, sont prolongeables à l'intervalle $\Delta$.

$3^{*}$ La fonction $q(t)$ est continue dans l'intervalle $\Delta$.

THÉoRìme I. Si les hypothèses (H) et $\left(\mathrm{H}^{*}\right)$ sont vérifiées, il existe une solution de l'équation (1) définie dans l'intervalle $(-\infty, a)$ et satisfaisant pour $t \leqslant 0$ à la condition initiale $\omega(t)$.

Pour la démonstration nous appliquerons la méthode bien connue de Tonelli. Dans ce but remplaçons l'équation différentielle (1) par l'équation intégrale suivante, qui lui est équivalente en vertu du lemme:

$$
\begin{array}{lll}
\varphi(t)=\omega(t) & \text { pour } & t \leqslant 0, \\
\varphi(t)=\omega(0)+\int_{0}^{t}\left\{\int_{0}^{\infty} f[\tau, \varphi(\tau-s)] d r(\tau, s)\right\} d \tau+\int_{0}^{t} q(\tau) d \tau & \text { pour } & t \epsilon\langle 0, a\rangle
\end{array}
$$

et construisons une suite de fonctions continues $\left\{\varphi_{v}(t)\right\}$ en posant

$$
\begin{array}{llrl}
\varphi_{\nu}(t)=\omega(t) & \text { pour } t \leqslant 0, \\
\varphi_{\nu}(t)=\omega(0)+\int_{0}^{t_{\nu}}\left\{\int_{0}^{\infty}\left[f\left[\tau, \varphi_{\nu}(\tau-s)\right] d r(\tau, s)\right\} d \tau+\int_{0}^{t} q(\tau) d \tau\right. & \text { pour } t \epsilon\langle 0, a\rangle,
\end{array}
$$

où $t_{\mathrm{v}}=\max (0, t-1 / \nu), \quad \nu=1,2,3, \ldots$ 
$\Pi$ est évident que les fonctions ainsi définies existent et sont continues dans l'intervalle $(-\infty, a)$.

Nous allons montrer que les fonctions $\left\{\varphi_{\boldsymbol{v}}(t)\right\}$ sont bornées dans leur ensemble et également continues dańs l'intervalle $(-\infty, a\rangle$. Cela est évident pour $t \leqslant 0$. Supposons donc que $t \epsilon\langle 0, a\rangle$; alors, en vertu de l'hypothèse $1^{*}$ on aura:

$$
\left|f\left[t, \varphi_{v}(t-s)\right]\right| \leqslant \Phi\left[t,\left|\varphi_{v}(t-s)\right|\right] \leqslant \Phi\left[t, \Lambda_{v}(t-s)\right] \leqslant \Phi\left[t, \Lambda_{v}(t)\right],
$$

où $\Lambda_{\boldsymbol{v}}(t)=\sup _{u \leq t}\left|\varphi_{v}(u)\right|$. Done, en posant $\eta=|\omega(0)|+\left|\int_{0}^{a} q(\tau) d \tau\right|$, on obtient pour $u \leqslant t$ en raison de $(4)$ et des propriétés bien connues de l'intégrale de Stieltjes, les inégalités:

$$
\begin{aligned}
\left|\varphi_{v}(u)\right| & \leqslant \eta+\int_{0}^{u_{v}} \Phi\left[\tau, \Lambda_{\nu}(\tau)\right] \bigvee_{s=0}^{\infty} r(\tau, s) d \tau \leqslant \eta+V \int_{0}^{u} \Phi\left[\tau, \Lambda_{\nu}(\tau)\right] d \tau \\
& \leqslant \eta+V \int_{0}^{l} \Phi\left[\tau, \Lambda_{\nu}(\tau)\right] d \tau,
\end{aligned}
$$

où $u_{v}=\max (0, u-1 / v)$ et $t \epsilon \Delta$. Il en résulte pour $t \in \Delta$ l'inégalité intégrale

$$
\Lambda_{v}(t) \leqslant \eta \div V \int_{0}^{t} \Phi\left[\tau, \Lambda_{v}(\tau)\right] d \tau, \quad v=1,2,3, \ldots
$$

En vertu d'un théorème de $Z$. Opial [4] sur les inégalités intégrales on a

$$
\Lambda_{v}(t) \leqslant g(t, \eta), \quad v=1,2,3, \ldots, t \in \Delta,
$$

où $g(t, \eta)$ désigne l'intégrale supérieure à droite de l'équation auxiliaire

$$
y^{\prime}(t)=V \Phi[t, y(t)],
$$

définie, en raison de l'hypothèse $2^{*}$, dans l'intervalle $\Delta$ et satisfaisant à la condition initiale $g(0)=\eta$. De l'inégalité $(\check{0})$ il résulte immédiatement que les fonctions $\left\{\varphi_{\nu}(t)\right\}$ sont bornées dans leur ensemble dans l'intervalle $\Delta$. D'où, en tenant compte de l'hypothèse $2^{\circ}$, on conclut qu'il existe un nombre $L>0$ tel que pour $t \epsilon(-\infty, a>$ sont vérifiées les inégalités

$$
\left|\varphi_{v}(t)\right| \leqslant L, \quad v=1,2,3, \ldots
$$

Supposons maintenant que les nombres $t$ et $t+h$ appartiennent à l'intervalle 4 ; des relations (4), ( 7 ) et de l'hypothèse $1^{*}$ il suit que

$$
\left|\varphi_{v}(t+h)-\varphi_{v}(t)\right| \leqslant \int_{t_{v}}^{t_{v}+h} \Phi(\tau, L) \bigvee_{s=0}^{\infty} r(\tau, s) d \tau \leqslant M V|h|,
$$


où $M$ est un nombre positif convenablement choisi, ce qui prouve que les fonctions $\{\varphi,(t)\}$ sont également continues dans l'intervalle $\Delta$.

En vertu du théorème d'Arzelà il existe donc une suite partielle de la suite $\left\{\varphi_{v}(t)\right\}$ uniformément convergente dans l'intervalle $(-\infty, a)$ vers une fonction $\varphi(t)$. Admettons, pour simplifier, qu'il en soit ainsi de la suite $\left\{\varphi_{v}(t)\right\}$ entière:

$$
\lim _{v \rightarrow \infty} \varphi_{v}(t) \Rightarrow \varphi(t) .
$$

Il est évident que pour $t \leqslant 0$ on a $\varphi(t)=\omega(t)$.

Pour prourer que la fonction $\varphi(t)$ satisfait à l'équation intégrale (3), on trouve pour $t \in \Delta$ une limitation de la différence

$$
R_{v}(t)=\left|\varphi_{v}(t)-\omega(0)-\int_{0}^{t}\left\{\int_{0}^{\infty} f[\tau, \varphi(\tau-s)] d r(\tau, s)\right\} d \tau-\int_{0}^{t} q(\tau) d \tau\right| \leqslant \sum_{i=1}^{2} A_{i}^{\nu}
$$

où

$$
\begin{aligned}
& A_{1}^{v}=\left|\int_{t_{v}}^{\ell}\left\{\int_{0}^{\infty} f\left[\tau, \varphi_{\nu}(\tau-s)\right] d r(\tau, s)\right\} d \tau\right|, \\
& A_{2}^{v}=\left|\int_{0}^{\ell}\left\{\int_{0}^{\infty}\left(f\left[\tau, \varphi_{\nu}(\tau-s)\right]-f[\tau, \varphi(\tau-s)]\right) d r(\tau, s)\right\} d \tau\right| .
\end{aligned}
$$

De l'hypothèse $1^{\circ}$ et des inégalités ( 7 ) on conclut qu'il existe un nombre positif $M$ tel que pour $t \in \Delta$ a lieu l'inégalité

$$
\left|f\left[t, \varphi_{\nu}(t-s)\right]\right| \leqslant M .
$$

On a done l'inégalité

d'oú il résulte que

$$
A_{\mathbf{1}}^{\boldsymbol{v}} \leqslant M V / \boldsymbol{\nu},
$$

$$
\lim _{\nu \rightarrow \infty} A_{1}^{\nu}=0 .
$$

Afin de trouver une limitation pour la fonction $A_{2}^{v}$ remarquons que, d'après (8) et l'hypothèse $1^{\circ}$, on a pour $v$ assez grands et $t \in \Delta$ l'inégalité

$$
\left|f\left[t, \varphi_{\nu}(t-s)\right]-f[t, \varphi(t-s)]\right|<\varepsilon,
$$

où $\varepsilon$ est un nombre positif aussi petit que l'on reut. On a donc alors l'inégalité $A_{2}^{v}<V a \varepsilon$, ce qui signifie que

$$
\lim _{v \rightarrow \infty} A_{2}^{v}=0 \text {. }
$$

La démonstration du théorème I est ainsi achevée. 
3. Considérons maintenant deux équations différentielles à argument retardé de mêmes noyaux:

$$
\begin{array}{rlr}
\varphi_{i}(t) & =\omega_{i}(t) & \text { pour } \quad t \leqslant 0, \\
\varphi_{i}^{\prime}(t) & =\int_{0}^{\infty} f_{i}\left[t, \varphi_{i}(t-s)\right] d r(t, s)+q_{i}(t) & \text { pour } \quad t \in \Delta, \\
& & i=1,2,
\end{array}
$$

et prenons comme terme de comparaison l'équation différentielle ordinaire:

$$
y^{\prime}(t)=V \Phi[t, y(t)]+\gamma(t), \quad t \in \Delta,
$$

où $\gamma(t)=\left|q_{1}(t)-q_{2}(t)\right|$ et le nombre $V$ est défini dans l'hypothèse $4^{\circ}$.

Supposons réalisées les hypothèses suivantes:

HYPOTHÈSES $\left(\mathrm{H}^{* *}\right)$. $\mathbf{1}^{* *}$ Les hypothèses $(\mathrm{H})$ et $2^{*}\left(\mathrm{H}^{*}\right)$ sont vérifiées.

$2^{* *}$ Pour tout couple de points $(t, \bar{x})$ et $(t, \overline{\bar{x}})$ du domaine $D$ on a les inégalités $\left|f_{1}(t, \bar{x})-f_{2}(t, \overline{\bar{x}})\right| \leqslant \Phi(t,|\bar{x}-\bar{x}|)$, la fonction $\Phi(t, y)$ ayant toutes les propriétés admises dans l'hypothèse $1^{*}\left(\mathrm{H}^{*}\right)$.

$3^{* *}$ Les fonctions $q_{i}(t)$ sont continues dans l'intervalle $\Delta, i=1,2$.

Désignons par $g(t, \eta)$ l'intégrale supérieure de l'équation (10), définie dans l'intervalle $\Delta$ et satisfaisant à la condition initiale $g(0)=\eta$, et $\operatorname{par} \varphi_{i}(t), i=1,2$, les intégrales des équations correspondantes (9), définies dans l'intervalle $(-\infty, a)$.

Nous pouvons maintenant énoncer le théorème suivant:

THÉORÈme II. Si les hypothèses $\left(\mathrm{H}^{* *}\right)$ sont vérifiées et si $\varphi_{i}(t)(i=1,2)$ sont les intégrales des équations correspondantes (9), définies dans l'intervalle $(-\infty, a\rangle$, ces intégrales satisfont dans l'intervalle $\Delta$ aux inégalités

$$
\left|\varphi_{1}(t)-\varphi_{2}(t)\right| \leqslant g(t, \eta), \quad \text { ò̀ } \quad \eta=\sup _{t \leqslant 0}\left|\omega_{1}(t)-\omega_{2}(t)\right| .
$$

Démonstration. Posons $\lambda(t)=\left|\varphi_{1}(t)-\varphi_{2}(t)\right|, t \epsilon(-\infty, a\rangle$. On voit aisément qu'on a, en raison des hypothèses $\left(\mathbf{H}^{* *}\right)$, l'inégalité

$$
\lambda(u) \leqslant \eta+\int_{0}^{u}\left\{\int_{0}^{\infty} \Phi[\tau, \lambda(\tau-s)] d r(\tau, s)+\gamma(\tau)\right\} d \tau, \quad u \in \Delta,
$$

dont on déduit sans difficulté pour $t \epsilon \Delta$ l'inégalité intégrale

$$
\Lambda(t) \leqslant \eta+\int_{0}^{t}\{V \Phi[\tau, \Lambda(\tau)]+\gamma(\tau)\} d \tau,
$$

où $\Lambda(t)=\sup _{u \leq i} \lambda(u)$. La conclusion de notre théorème résulte alors immédiatement de la proposition sur les inégalités intégrales citée plus haut. 
Dans le cas où $\omega_{1}(t) \equiv \omega_{2}(t), \quad f_{1}(t, x) \equiv f_{2}(t, x)$ et $q_{1}(t) \equiv q_{2}(t)$, le théorème II permet de trouver une limitation pour la différence de deux intégrales de l'équation (1) qui satisfont à la même condition initiale et il généralise un théorème de $\mathrm{E}$. Bompiani [2].

Du théorème II on déduit immédiatement un critère d'unicité pour les solutions de l'équation différentielle (1). Nous l'énonçons sous forme du théorème suivant:

THÉonÈmE III. Si les hypothèses $\left(\mathrm{H}^{* *}\right)$ sont vérifiées et si l'intégrale supérieure de l'équation différentielle ordinaive (6) satisfait pour $t \in \Delta$ à la condition $g(t, 0) \equiv 0$, l'équation différentielle (1) admet exactement une solution, définie dans l'intervalle $(-\infty, a>$ et satisfaisant pour $t \leqslant 0$ à la condition initiale $\omega(t)$.

Remarque 1. En choisissant convenablement la fonction $\Phi(t, y)$ on peut obtenir certains critères d'unicité des solutions de l'équation (1), connus pour les équations différentielles ordinaires.

Remarque 2. Si les hypothèses du théorème II sont réalisées et si, de plus, l'intégrale supérieure $g(t, \eta)$ de l'équation différentielle (6) dépend continûment, pour $\eta$ positifs assez petits, des conditions initiales, alors la solution de l'équation différentielle à argument retardé (1) dépend continûment des fonctions initiales $\omega(t)$.

Remarque 3. Les théorèmes que nous venons d'établir s'étendent sans modification essentielle au cas où la fonction $f(t, x)$ admet ses valeurs dans l'espace euclidien à $n$ dimensions $E_{n}$ et est définie et continue pour $t \in \Delta, x \in E_{n}$.

\section{Travaux cités}

[1] A. Bielecki, et M. Maksym, Sur une généralisation d'un théorème de A. D. Myshkis concernant un système d'équations différentielles ordinaires à argument retardé, Folia Soc. Sci. Lublinensis 2 (1962), p. 74-78.

[2] E. Bompiani, Un teorema di confronto ed un teorema di unicità per l'equazione differenziale $y^{\prime}=f(x, y)$, Rend. B. Acc. Naz. dei Lincei (6), I (1925), p. 298-302.

[3] А. D. Мышки (А. D. Myszkis), Линейные дифференциальные уравнения с запаздываюичм араументом, Moskwa-Leningrad 1951.

[4] Z. O pial, Sur un système d'inégalités intégrales, Ann. Polon. Math. 3 (1957), p. 200-209. 